$\begin{array}{cl}\begin{array}{c}\text { Revue } \\ \text { de } / \text { histoire } \\ \text { des religions }\end{array} & \text { Revue de l'histoire des religions } \\ & \begin{array}{l}1 \mid 2014 \\ \text { Varia }\end{array}\end{array}$

\title{
Autour de l'Exode : monothéisme, différence et violence
}

Around Exodus : monotheism, difference and violence

Jan Assmann

Traducteur : Jacob Schmutz

\section{OpenEdition}

\section{Journals}

Édition électronique

URL : http://journals.openedition.org/rhr/8189

DOI : $10.4000 /$ rhr.8189

ISSN : $2105-2573$

\section{Éditeur}

Armand Colin

\section{Édition imprimée}

Date de publication : 1 mars 2014

Pagination : 5-26

ISBN : 978-2200929107

ISSN : 0035-1423

\section{Référence électronique}

Jan Assmann, «Autour de l'Exode : monothéisme, différence et violence », Revue de l'histoire des religions [En ligne], 1 | 2014, mis en ligne le 01 mars 2017, consulté le 19 avril 2019. URL : http:// journals.openedition.org/rhr/8189; DOI : 10.4000/rhr.8189 


\section{JAN ASSMANN \\ Université de Constance \\ Autour de l'Exode : monothéisme, différence et violence}

Le but de l'article est de réviser la notion de la «distinction mosaïque». À y regarder de plus près, le livre de l'Exode comporte non pas une, mais trois distinctions "mosaïques», dont aucune ne porte sur la vérité et la non-vérité, mais plutôt sur la servitude et la liberté, le peuple élu et les autres, l'ami et l'ennemi. Le monothéisme de la vérité n'apparaît que plus tard, avec le Deutéro-Isaïe, Jérémie et d'autres prophètes plus tardifs et post-exiliques. La religion fausse, à savoir l'idolâtrie, y est objet de dédain et de dérision, mais pas de persécution violente: les autres dieux n'existent simplement pas. Cette nouvelle idée ne supplante pourtant jamais le monothéisme de la fidélité, qui reste dominant dans les trois religions abrahamiques.

\section{Around Exodus: monotheism, difference and violence}

The article aims at a revision of the notion of «Mosaic distinction». A closer look at the book of Exodus shows not one, but three "Mosaic» distinctions, which are not about truth and untruth, but about slavery and freedom, the chosen people and the rest, friend and foe. The monotheism of truth comes only later, with Deutero-Isaiah, Jeremiah and other late- and post-exilic prophets. The false religion, i.e. idolatry, is an object of scorn and derision, but not of violent persecution. The other gods are deemed inexistent. This new idea, however, never supersedes the monotheism of faithfulness that remains dominant in the three Abrahamic religions. 
D'où vient la force polarisatrice caractéristique des religions monothéistes ? ${ }^{1}$ Elles seules ont cette propriété de pouvoir diviser le monde en Juifs et Gentils, chrétiens et païens, musulmans et infidèles, et même au sein d'elles-mêmes, entre juifs et samaritains, Église d'Occident et Église d'Orient, catholiques et protestants, sunnites et chiites, etc. Ces divisions ont souvent dégénéré en intolérance, en persécution et en violence: sinon dans le judaïsme rabbinique, en tout cas dans le christianisme et dans l'islam. Le problème est actuel, parce que nous sommes jusqu'aujourd'hui confrontés à cet antagonisme religieux et à ses expressions violentes ; mais la question de savoir d'où il vient est une question d'origine et s'adresse dès lors à la Bible hébraïque, qui constitue le fondement des trois religions dites abrahamiques, mais surtout du judaïsme et du christianisme. Personne ne contestera que la Bible est remplie de scènes de violence religieuse, à commencer par les 3000 hommes que Moïse a laissé exécuter après la danse autour du veau d'or jusqu'aux victimes de la réforme cultuelle de Josias et jusqu'à la dissolution des mariages mixtes sous Ezra et Néhémie, en passant par Zimri et son amante madianite, que Pinhas a assassinés dans leur couche après la fête pour Ba'al Pe'or, ou encore les 400 prêtres de Ba'al exécutés par Élias et le roi des Amalécites Agag, dépecé par Samuel. D’où vient cette violence, et que doit-elle signifier aujourd'hui?

\section{LES DISTINCTIONS «MOSAÏQUES» ET L'ORIGINE DE L'INTOLÉRANCE}

Dans mes ouvrages Moïse l'Égyptien et Le prix du monothéisme², j'ai rapporté cette force polarisatrice à un principe, que j'ai appelé la «distinction mosaïque». Ma thèse était que le monothéisme a

1. Les réflexions exposées ici ont fait l'objet d'une conférence que j'ai donnée au Laboratoire d'études sur les monothéismes (LEM - UMR 8584) du CNRS, à Paris, le 28 mai 2013 dans le cadre du projet «Revisiter les monothéismes », dirigé par Constantinos Macris en collaboration avec Anna van den Kerchove et Joëlle Soler.

2. Jan Assmann, Moïse l'Égyptien: un essai d'histoire de la mémoire, trad. L. Bernardi, Paris, Aubier, 2001, réimpr. Paris, Flammarion («Champs», 547), 2003 (original anglais 1997, allemand 1998); Id., Le prix du monothéisme, trad. L. Bernardi, Paris, Aubier, 2007 (original allemand 2003). 
été le premier à avoir introduit la distinction entre le vrai et le faux dans l'espace de la religion. Ce n'est qu'à partir de ce moment qu'il devenait possible de faire la différence entre la religion vraie et des religions fausses, entre le Dieu vrai et des faux dieux, et donc également de se convertir d'une religion fausse à la religion vraie, et inversement d'apostasier la vraie religion et de tomber dans une fausse. Mon concept de «distinction mosaïque»a provoqué beaucoup d'indignation dans les cercles théologiques et on est allé jusqu'à m'accuser d'antisémitisme ${ }^{3}$. Les objections les plus importantes se laissent résumer sous la forme de deux arguments: premièrement, le souci de Moïse - quelle que soit la façon dont nous voulons nous le représenter, soit comme une figure historique, soit comme une figure simplement littéraire - n'était pas la vérité, mais la libération. La distinction qui est au fondement du mythe de l'Exode qui a mené les Juifs hors d'Égypte repose sur l'opposition entre la servitude et la liberté, et non pas sur celle entre la vérité et la non-vérité. Il en découle - et c'est le deuxième argument que le reproche d'intolérance s'écroule également. L'intolérance appartient au registre de la vérité, et non à celui de la liberté. Mais cela permet surtout de rejeter le reproche que l'idée monothéiste serait associée à la violence: bien au contraire, le monothéisme aurait rendu le monde plus paisible, dans la mesure où il aurait préparé la fin des luttes polythéistes entre les dieux et de son reflet dans les guerres terrestres. En outre, comment pourrait-il y avoir le moindre rapport entre la vérité et la violence? L'exercice de la violence repose sur la distinction entre l'ami et l'ennemi, et non sur celle entre le vrai et le faux.

Ces objections sont justifiées et nous invitent à repenser une fois de plus la question de la distinction et de la violence. De fait, les concepts «Moïse», «mosaïque» et «distinction entre le vrai et le faux» ne se laissent pas combiner facilement. Le nom de Moïse est associé au mythe de l'Exode, un mythe qui associe la sortie, la révélation, l'élection et l'alliance avec Dieu ainsi que la terre promise. Il n'est pas ici question de vérité, mais de plusieurs distinctions fondamentales qui se déploient dans les deux parties

3. Richard Wolin, «Biblical Blame Shift: Is the Egyptologist Jan Assmann Fueling Anti-Semitism?», The Chronicle of Higher Education, 15 avril 2013, http://chronicle.com/article/Biblical-Blame-Shift/138457/. 
du livre de l'Exode, dans les récits de la sortie d'Égypte et de la constitution de l'alliance dans le Sinaï. La distinction qui est au fondement de la première partie est très clairement celle entre la servitude et la liberté. Dans la deuxième partie, il s'agit en revanche d'obligation, et la connexion qui relie ces deux parties repose sur l'idée que cette obligation signifie en réalité la liberté. La loi à laquelle s'obligent les hommes libérés les rend libres de toute forme d'oppression humaine.

Deux autres nouvelles distinctions entrent alors en jeu, et elles n'ont toutes les deux rien à voir avec le vrai et le faux. La première distingue entre l'intérieur et l'extérieur, l'appartenance et la nonappartenance à l'alliance, l'endo- et l'exosphère. Cette distinction résulte de l'idée d'alliance et d'élection. Dieu ne conclut pas cette alliance avec le monde et avec l'humanité, mais avec les enfants d'Israël, qu'il a choisis et fait sortir de l'Égypte comme son propre peuple à lui. Le livre de l'Exode dit expressément qu'un grand nombre d'autres gens se sont associées aux Israélites qui sortaient de l'Égypte (Ex 12, 38; voir aussi Nombres 11, 4) : il faut en déduire que la descendance n'est pas le critère de l'appartenance à l'alliance, mais seulement et uniquement le corps des lois auxquelles s'obligent par un serment les hommes ainsi libérés. C'est par l'alliance et l'élection que le monde se divise entre Israël et les nations. Cette distinction, il faut le souligner expressément, n'a rien à voir avec une quelconque polarisation, avec l'intolérance et la violence, et certainement rien non plus avec la distinction entre l'ami et l'ennemi. Dieu se soucie également des autres peuples, mais il poursuit un projet particulier pour Israël. En revanche, au sein de l'alliance, dans l'endosphère, c'est une autre distinction qui prévaut, et cette dernière est très explicitement la distinction entre l'ami et l'ennemi. Elle constitue le fondement de l'interdiction d'honorer d'autres dieux ou d'adorer les images, et se situe dès lors à un endroit tout à fait central:

Tu ne te prosterneras pas devant ces dieux ni ne les serviras. Car moi, Yahvé, ton Dieu, je suis un Dieu jaloux, qui punis la faute des pères sur les enfants, les petits-enfants et les arrière-petits-enfants, pour ceux qui me haïssent, mais qui fais grâce à des milliers, pour ceux qui m'aiment et gardent mes commandements (Dt 5, 9-10 = Ex 20,

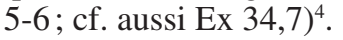

4. Toutes les citations bibliques sont traduites selon la version de l'École biblique de Jérusalem (N.d.T.). 
Quand bien même Dieu établit le rapport entre sa jalousie susceptible de colère ( $q$ in'ah) et sa miséricorde pleine de compassion (hesed) à trois contre mille, l'importance de cette distinction n'en est pas moins clairement établie et toujours rappelée dans de nombreux autres récits et épisodes. Ce que Dieu exige de son peuple est la fidélité, une fidélité inconditionnelle et absolue, qui s'accomplit dans l'observation de ses commandements et interdits. Or, on ne peut être fidèle ou infidèle que là où il $\mathrm{y}$ a des alternatives. Il est impossible d'être infidèle à l'égard du créateur, car il est impossible d'échapper à la condition de créature; en revanche, il est possible d'être infidèle à l'égard du libérateur, par exemple en retournant à la servitude égyptienne, en le quittant pour d'autres dieux ou bien encore en brisant les lois. Les lois ne sont ni vraies ni fausses, mais seulement contraignantes ou obligeantes. C'est la raison pour laquelle le mythe de l'Exode ne parle jamais de Dieu comme du créateur du ciel et de la terre, mais seulement comme du libérateur, comme «celui qui t'a fait sortir de l'Égypte, la maison de l'esclavage ». Ce Dieu est celui qui distingue entre l'ami et l'ennemi, et qui accorde à l'un sa miséricorde et à l' autre sa colère. La colère et la crainte appartiennent à cette alliance, au même titre que l'amour et la miséricorde, quand bien même l'amour est plusieurs centaines de fois plus fort que la colère. Lactance, qui a consacré un écrit à la colère de Dieu, a donné une véritable formulation de ce principe: religio esse non potest ubi metus nullus est ${ }^{5}$, «il ne peut pas y avoir de religion là où il n'y a pas de peur». Cela vaut non seulement pour la religion biblique, mais aussi pour toute autre religion. Les autres peuples aussi ont aimé et craint leurs dieux. La spécificité du monothéisme biblique repose dans l'idée de l'alliance et de la fidélité. C'est en cela qu'une forme totalement nouvelle de religion a été inventée, fondée sur l'obligation et l'appartenance.

\section{LE MONOTHÉISME DE LA FIDÉLITÉ ET LA GUERRE SAINTE}

Cette nouvelle religion qui repose sur le concept de fidélité a trouvé dans le mythe de l'Exode son expression contraignante.

5. L. Caelii Firmiani Lactantii, De Ira Dei Liber, 11.15 (Laktanz, Vom Zorne Gottes, eingel., hrsg., übertragen u. erläutert von H. Kraft und A. Wlosok, Darmstadt 1957). 
Le prophète Osée, qui est actif au $\mathrm{VIII}^{\mathrm{e}}$ siècle au moment de l'effondrement du royaume du Nord face aux Assyriens, et chez qui ce mythe apparaitt pour la première fois, a décrit cette fidélité en utilisant les images de la filiation, des fiançailles et du mariage, et a interprété l'adoration d'autres dieux comme une forme d'adultère et de fornication. Le Deutéronome, dont la version primitive est née une centaine d'années plus tard, a formulé cette fidélité d'après le modèle des contrats de vassalité et des serments de loyauté assyriens, en transférant le principe politique de la loyauté auquel s'obligent les vassaux et les sujets par rapport à leur souverain à la relation religieuse entre Dieu et le peuple de l'alliance ${ }^{6}$. Les distinctions entre fidélité et apostasie ainsi qu'entre ami et ennemi remontent à cette origine proprement politique du modèle. À travers sa réinterprétation (ou ce que j'appelle son «transfert», Umbuchung) au rapport entre l'homme et Dieu, ce modèle subit toutefois trois modifications significatives. Premièrement, le lien d'obligation dont il est question est indissolublement associé au concept de libération à travers le mythe de l'Exode. On ne comprend le sens des lois que si l'on se rappelle l'esclavage en Égypte. Deuxièmement, l'alliance avec Dieu n'est pas octroyée de façon autoritaire sous la forme d'un contrat de vassalité, mais elle est conclue de façon volontaire et est également toujours renouvelée de façon volontaire ; une grande importance est accordée à cet aspect. Troisièmement, les aspects de la miséricorde, bonté, patience et compassion jouent ici un tout autre rôle que dans le modèle politique. De toute évidence, pourtant, la distinction entre ami et ennemi provient de cette source, et avec elle le problème de l'intolérance et de la violence.

Dans le livre de la Genèse, il n'est pas question d'ami ni d'ennemi, et encore moins de colère ou de jalousie de Dieu, même s'il y est souvent question de violence punitive, à commencer par l'expulsion du paradis et le déluge jusqu'à la destruction de Sodome et Gomorrhe, en passant par la confusion des langues - durant toutes ces interventions violentes, jamais n'éclate la

6. Eckart Otto, Das Deuteronomium. Politische Theologie und Rechtsreform in Juda und Assyrien, Berlin / New York, De Gruyter, 1999; Hans Ulrich Steymans, Deuteronomium 28 und die adê zur Thronfolgeregelung Asarhaddons. Segen und Fluch im Alten Orient und in Israel, Fribourg (Suisse), Universitätsverlag / Göttingen, Vandenhoeck \& Ruprecht, 1995. 
colère de Dieu? ${ }^{7}$ Cela se poursuit de la même manière dans le livre de l'Exode. Derrière les dix plaies que Dieu fait tomber sur l'Égypte, on ne trouve pas de colère enflammée, mais le souhait d'envoyer des signes et de démontrer sa puissance. La colère et la jalousie appartiennent seulement à la sémantique de l'alliance qui est nouée dans le Sinaï, et le récit du veau d'or a pour objet de rendre cela clair et évident. C'est ainsi que le voyait déjà Lactance, en rapportant dans son écrit De ira Dei la colère de Dieu à son imperium, c'est-à-dire à son rôle comme maître de l'alliance et non à son essence. C'est à cet imperium qu'appartient la distinction entre l'ami et l'ennemi. Elle appartient à un Dieu qui ne se contente pas de veiller à l'observance des contrats et des lois, comme le font beaucoup d'autres dieux - par exemple Rê en Égypte, Schamasch en Babylonie, Zeus en Grèce, Mithra en Perse, Varuna en Inde, le Ba'alberît à Canaan, etc. Bien au contraire, et c'est cela qui est l'extraordinaire nouveauté de cette religion, c'est un Dieu qui édicte lui-même ces lois et contrats. Tel est le libérateur de la servitude égyptienne dont il est question dans l'Exode. Le Dieu de la Genèse, en revanche, est le créateur du ciel et de la terre.

Nous ne devons pas en déduire pour autant que le monothéisme de la fidélité ait été la religion d'Israël aux $\mathrm{VIII}^{\mathrm{e}}-\mathrm{VI}^{\mathrm{e}}$ siècles. Il constituait la cause d'une minorité oppositionnelle, en particulier des prophètes, dont le message a rencontré une résistance massive à la cour et apparemment aussi dans de larges sections du peuple: «ils jetèrent ta Loi derrière leur dos, ils tuèrent les prophètes qui les avertissaient pour te les ramener et commirent de grands blasphèmes » (Néhémie 9, 26; comparer avec Luc 13, 34: «Jérusalem, Jérusalem, toi qui tues les prophètes et lapides ceux qui te sont envoyés»). Ce n'est qu'au moment de l'exil, lorsque le malheur prophétisé s'était abattu sur le peuple, que ce courant s'est imposé.

Bien avant que le document sacerdotal n'ait fait converger les livres de la Genèse et de l'Exode vers la fin du vi ${ }^{\mathrm{e}}$ siècle, et que des rédacteurs plus tardifs ne les aient associés au Deutéronome, pour ainsi constituer le canon à cinq livres de la Torah, ils ont

7. Cette importante observation a été faite par Claus Westermann, «Boten des Zorns. Der Begriff des Zornes Gottes in der Prophetie», Die Botschaft und die Boten. Festschrift für Hans Walter Wolff zum 70. Geburtstag, éd. Jörg JeremiasLothar Perlitt, Neukirchen-Vluyn, Neukirchener Verlag, 1981, p. 147-156. 
néanmoins pu auparavant mener une vie littéraire comme mythes alternatifs de l'origine d'Israël ${ }^{8}$. Les deux mythes rapportent Israël à une origine différente. Le mythe des patriarches de la Genèse voit Dieu appeler Abraham depuis la Mésopotamie pour le conduire jusqu'à Canaan et forger avec lui une alliance et lui promettre, ainsi qu'à son innombrable descendance, cette terre comme propriété; dans le mythe de l'Exode en revanche, tel qu'il est exprimé par Osée, Dieu appelle son fils - à savoir les enfants d'Israël devenus un peuple - de l'Égypte et forge avec lui une alliance dans le Sinaï par l'entremise de Moïse. Dans l'alliance d'Abraham, il n'est question que de descendance et le signe de la circoncision y joue le rôle d'un critère d'appartenance; il n'y est jamais question de fidélité, mais seulement de confiance (emunah, le concept qui sera ensuite, dans un contexte chrétien, interprété comme foi), à savoir la confiance en la promesse. Dans l'alliance mosaïque en revanche, c'est le corpus des commandements et des interdits qui constitue le critère de l'appartenance, et la fidélité devient le concept central. Dans l'horizon de la Genèse et du mythe des patriarches, il n'est jamais question d'autres dieux; bien au contraire, le roi cananéen Melkisédeq de Salem constate explicitement que son Dieu et celui d'Abraham sont le même, à savoir le créateur du ciel et de la terre (Gn 14, 18-20). En revanche, les autres dieux et l'interdiction de les honorer forment le fondement de l'alliance mosaïque.

Le document sacerdotal a unifié ces deux mythes originels sous la forme d'une œuvre historique englobante, en présentant le mythe des patriarches comme la préhistoire du mythe de l'Exode, puis en les associant l'un à l'autre par le récit de Joseph. En suivant le modèle des listes royales babyloniennes et égyptiennes, cette double histoire de l'origine a été complétée par une préhistoire et ramenée jusqu'à la création du monde. Les deux récits de l'origine ont en commun l'insistance sur l'origine étrangère, l'allochtonie. C'est ainsi qu'Israël se définit comme étranger dans le pays de Canaan et se distingue fortement des autres habitants du pays. Le mythe des patriarches présente ce rapport d'invité ou d'étranger sous une

8. Voir sur ce point Konrad Schmid, Erzväter und Exodus. Untersuchungen zur doppelten Begründung der Ursprünge Israels innerhalb der Geschichtsbücher des Alten Testaments, Neukirchen-Vluyn, Neukirchener Verlag, 1999 (trad. angl. J. D. Nogalski, Genesis and the Moses story. Israel's dual origins in the Hebrew Bible, Winona Lake [Indiana], Eisenbrauns, 2010). 
forme sympathique: le pays n'est pas conquis, mais acheté, des contrats sont établis avec ses habitants originaires, et on vit en bonne entente sur la base d'une tolérance mutuelle. Le mythe de l'Exode, en revanche, détermine le rapport entre immigrants et habitants autochtones sous les traits d'une guerre sainte. Aucun contrat ne doit être conclu, et les hommes ne doivent pas être épargnés. Le seul traitement possible pour les Cananéens est l'extermination et la dispersion, pour la bonne et simple raison que ces peuples pourraient séduire les Israélites et les convertir à leurs coutumes, que Dieu a pourtant en haine. Autant le mythe des patriarches est placé sous le signe de la tolérance et de l'entente entre les peuples, autant le mythe de l'Exode est placé sous le signe de l'abomination et de la persécution. D'abord, c'est l'Égypte d'où sortent les libérés qui est présentée sous les couleurs les plus noires d'une oppression qui n'hésite pas à employer jusqu'à des mesures génocidaires, et ensuite, le peuple de Dieu se voit enjoint de chasser et d'exterminer avec la dureté la plus intransigeante les peuples vivant à Canaan. Après qu'ils aient été des victimes en Égypte, l'alliance divine leur impose la tâche sainte de devenir à leur tour des meurtriers à Canaan.

Les deux récits de l'origine, qu'il s'agisse du mythe pacifique et inclusif des patriarches ou bien du mythe agressif et exclusif de l'Exode, ont en commun le motif de l'allochtonie, à savoir la provenance d'un pays étranger. D'un point de vue archéologique comme anthropologique, cela ne se laisse toutefois confirmer d'aucune manière. Du point de vue de leur culture matérielle et de leurs signes distinctifs anthropologiques, les anciens Hébreux ne se distinguaient de toute évidence en rien des autres habitants du pays ${ }^{9}$. Nous avons donc affaire ici à un phénomène de sémantique culturelle et non de souvenir historique. Le sens profond de cette sémantique de l'allochtonie, de l'immigration depuis une terre étrangère, est le motif de la séparation, ou encore, pour l'exprimer avec la signification la plus forte et la plus prégnante de la séparation, la sacralisation. Telle est la signification propre de la

9. Israel Finkelstein - Neil Asher Silverman, La Bible dévoilée. Les nouvelles révélations de l'archéologie, trad. P. Ghirardi, Paris, Bayard, 2002, réimpr. Paris, Flammarion («Folio. Histoire», 127), 2004; Othmar Keel, Die Geschichte Jerusalems und die Entstehung des Monotheismus, 2 vols, Göttingen, Vandenhoeck \& Ruprecht, 2007. 
distinction qui est établie à travers l'alliance et l'élection, et elle appartient à tous les deux mythes, à celui des patriarches comme à celui de l'Exode. Avec l'acte de l'élection et de l'établissement de l'alliance, les partenaires qui ne sont dans le livre de la Genèse qu'un seul avec sa famille et ses descendants, deviennent un peuple entier dans l'Exode, qui se sépare toutefois du reste de l'humanité et de tous les autres habitants du pays. Dieu appelle les enfants d'Israël à rejoindre l'alliance avec les mots suivants: «Maintenant, si vous écoutez ma voix et gardez mon alliance, je vous tiendrai pour mon bien propre parmi tous les peuples, car toute la terre est à moi. Je vous tiendrai pour un royaume de prêtres, une nation sainte» (Ex 19, 5-6). C'est par l'alliance qu'Israël devient un «peuple qui habite à part, il n'est pas rangé parmi les nations » (Nombres 23,9).

Telle est la véritable «distinction mosaïque», et celle-ci n'a rien à voir avec la distinction entre la religion vraie et la religion fausse, du Dieu vrai et des dieux faux. Elle n'a également rien à voir avec la distinction entre l'ami et l'ennemi. Si nous nous sommes demandé d'où venait la force polarisatrice des religions monothéistes, qui se distinguent toujours des autres - païens, infidèles, idolâtres, polythéistes, etc. -, alors nous devons souligner ici que cette énergie antagoniste ne provient pas de la distinction mosaïque entre Israël et les nations. La distinction entre l'ami et l'ennemi, telle qu'elle est formulée dans le premier (ou dans le premier et le deuxième) commandement, ne vaut qu'au sein de l'alliance. Les autres ne sont pas des ennemis. À une exception près, à savoir les sept peuples qui sont établis dans la terre promise. Avec eux prévaut la sémantique de la guerre sainte, pour laquelle la sémantique entre l'ami et l'ennemi valable au sein de l'alliance peut être transférée à la relation extérieure entre le peuple de l'alliance et ses voisins. Les recommandations à cet égard sont sans équivoque. Dans le livre du Deutéronome, il est dit:

Lorsque Yahvé ton Dieu t'aura fait entrer dans le pays dont tu vas prendre possession, des nations nombreuses tomberont devant toi: les Hittites, les Girgashites, les Amorites, les Cananéens, les Perizzites, les Hivvites et les Jébuséens, sept nations plus nombreuses et plus puissantes que toi. Yahvé ton Dieu te les livrera et tu les battras. Tu les dévoueras par anathème. Tu ne concluras pas d'alliance avec elles, tu ne leur feras pas grâce. Tu ne contracteras pas de mariage avec elles, tu ne donneras pas ta fille à leur fils, ni ne prendras leur fille pour ton fils. Car ton fils serait détourné de me suivre; il servirait d'autres dieux; 
et la colère de Yahvé s'enflammerait contre vous et il t'exterminerait promptement. Mais voici comment vous devrez agir à leur égard: vous démolirez leurs autels, vous briserez leurs stèles, vous couperez leurs pieux sacrés et vous brûlerez leurs idoles. Car tu es un peuple consacré à Yahvé ton Dieu; c'est toi que Yahvé ton Dieu a choisi pour son peuple à lui, parmi toutes les nations qui sont sur la terre (Dt 7, 1-6).

Et dans le livre de l'Exode:

Je fixerai tes frontières de la mer des Roseaux à la mer des Philistins, et du désert au Fleuve, car je livrerai entre vos mains les habitants du pays, et tu les chasseras devant toi. Tu ne feras pas alliance avec eux ni avec leurs dieux. Ils n'habiteront pas ton pays, de peur qu'ils ne te fassent pécher contre moi, car tu servirais leurs dieux et ce serait pour toi un piège (Ex 23, 31-33).

La sémantique de la guerre sainte n'a rien de spécifiquement biblique $^{10}$. On en trouve par exemple le concept dans l'inscription de la stèle du roi Mesha de Moab, qui date du milieu du IX ${ }^{\mathrm{e}}$ siècle ${ }^{11}$. La guerre sainte est une guerre d'extermination, au cours de laquelle tout butin est interdit, mais dans laquelle l'ensemble du butin et des victimes doivent être présentés en sacrifice à Dieu, au nom duquel la guerre est menée et dont le soutien est continuellement imploré. Il s'agit ici d'une monolâtrie occasionnelle et temporaire, valable uniquement pour l'état d'urgence, qui a très certainement joué un rôle important dans la formation du monothéisme biblique de la fidélité. Au chapitre XX du Deutéronome, il est prescrit que l'on peut mener une guerre normale contre des peuples ou des cités lointaines, et dont on peut prendre un butin; en revanche, face aux peuples et aux cités des Cananéens, il faut mener une guerre sainte.

Les trois distinctions qui sont établies à l'aide du récit dans le mythe de l'Exode et qui peuvent, pour cette raison, être à juste titre qualifiées de «mosaïques», sont donc celles entre la servitude et

10. Sa-Moon Kang, Divine War in the Old Testament and in the Ancient Near East, Berlin / New York, De Gruyter, 1989; Thomas von der Way, Göttergericht und "heiliger Krieg" im Alten Ägypten, Heidelberger Orientverlag ( Studien zur Archäologie und Geschichte Altägyptens», 4), 1992; Bernhard Lang, Buch der Kriege - Buch des Himmels. Kleine Schriften zur Exegese und Theologie, Louvain, Peeters, 2011.

11. «Die Inschrift des Königs Mesa von Moab», Texte aus der Umwelt des Alten Testaments, vol. 1: Rechts- und Wirtschaftsurkunden. Historischchronologische Texte, éd. Otto Kaiser, Gütersloh, Gütersloher Verl.-Haus Mohn, 1985, p. 646-650 ; Christian Molke, Der Text der Mescha-Stele und die biblische Geschichtsschreibung, Francfort/Main e.a., Peter Lang, 2006. 
la liberté ${ }^{12}$, entre l'alliance et le monde extérieur (en l'occurrence Israël et les nations) et enfin entre amis et ennemis de Dieu. Ces trois distinctions caractérisent la religion de l'Exode comme un monothéisme de la fidélité. Parmi ces trois distinctions «mosaïques », c'est la troisième seulement - celle entre les amis et les ennemis de Dieu - que l'on pourrait rapporter à notre question de l'origine de l'énergie polarisatrice et antagoniste du monothéisme. Elle est également la seule des trois distinctions qui trouve ses équivalences et probablement aussi son origine dans l'environnement d'Israël. Pour la distinction entre ami et ennemis au sein de l'alliance, c'està-dire entre les fidèles et les apostats, l'origine est à chercher dans le loyalisme étatique assyrien; pour la relation entre le peuple de Dieu et ses voisins immédiats, l'origine repose dans l'institution de la guerre sainte. Le loyalisme étatique assyrien est ainsi transposé à la relation avec Dieu et la monolâtrie occasionnelle et temporaire de la guerre sainte reçoit un caractère désormais durable.

Le caractère agressif et exclusiviste du livre de l'Exode ressort fortement lorsqu'on le compare avec la tendance pacifique et inclusiviste du livre de la Genèse. Il n'en demeure pas moins que l'histoire de la sortie d'Égypte est sans aucun doute l'histoire la plus formidable et la plus riche en conséquences que les hommes se soient jamais racontée. Les monothéismes juif, chrétien et islamique - dans lesquels se reconnaît aujourd'hui près de la moitié de l'humanité - n'existeraient pas sans la théologie de la libération, de l'alliance et de la promesse déployée dans le mythe de l'Exode. C'est ce mythe qui a permis au peuple juif de survivre aux catastrophes de la destruction du royaume du Nord par les Assyriens et 150 ans plus tard à celle du royaume du Sud par les Babyloniens, et qui leur a permis d'être le seul peuple de l'Antiquité à avoir survécu à travers les nombreux siècles de la diaspora jusqu'aujourd'hui. Tel est le cadre théologique auquel correspond le nom de Moïse: il n'est pas question ici de vérité, mais de fidélité. Dans les sciences religieuses, cette forme de monothéisme est en général désignée comme une «monolâtrie». La monolâtrie présuppose l'existence d'autres dieux et exige l'adoration exclusive d'un seul. Le mono-

12. «Liberté» n'est pourtant pas un mot biblique. La distinction que le récit de l'Exode établit est celle entre le service (avodah) du Pharaon et le service de Dieu. Le service de Dieu libère de tout esclavage humain. 
théisme en revanche conteste l'existence d'autres dieux. Cette terminologie n'est en fait pas très heureuse, car elle laisse supposer que la théologie de l'alliance du livre de l'Exode serait quelque chose de typique et de répandu. En réalité, elle constitue un fait unique et révolutionnaire, qui ne se trouve que dans la Bible. C'est pourquoi je préfère parler de monothéisme de la fidélité plutôt que de monolâtrie.

\section{LE MONOTHÉISME DE LA VÉRITÉ}

À côté de ce monothéisme de la fidélité, qui représente ce qu'il y a de spécifique et de nouveau dans la religion judéo-israélite, on trouve alors une forme tout à fait différente de monothéisme qui apparaît dans l'exil babylonien, très probablement sous influence babylonienne et perse. Dans cette nouvelle forme de monothéisme, les autres dieux n'existent plus, et je qualifierai cette forme du nom de «monothéisme de la vérité». L'unicité de Dieu n'est plus ici une question de fidélité, mais d'intelligence. Les autres dieux sont des fictions, des fétiches, des artefacts humains et des imaginations, c'est-à-dire des «idoles ». C'est ici que la distinction entre le «vrai» et le «non-vrai» entre en jeu et sépare l'espace de la religion entre celle qui est vraie et celles qui sont fausses, entre le seul et unique Dieu vrai et les nombreux dieux faux. Ce monothéisme de la vérité ne s'applique plus à l'unique qui nous a libéré de la servitude égyptienne, mais cette fois-ci au créateur du ciel et de la terre:

Ainsi parle Yahvé, roi d'Israël, Yahvé Sabaot, son rédempteur: Je suis le premier et je suis le dernier, à part moi, il n'y a pas de dieu (Is $44,6)$.

Ainsi parle Dieu dans le Deutéro-Isaïe. Les autres religions ne sont rien d'autre qu'une vaniteuse idolâtrie. Les idolâtres ne sont pas infidèles, mais des imbéciles totalement décervelés:

Ils ne savent pas, ils ne comprennent pas, car leurs yeux sont incapables de voir, et leur cœur de réfléchir; pas un ne rentre en luimême, pas un n'a la connaissance et l'intelligence (...) (Is 44, 18-19).

C'est à ce type de monothéisme contestant fondamentalement l'existence d'autres dieux qu'appartiennent par exemple le culte exclusiviste du soleil introduit par Akhénaton en Égypte ainsi 
que le monothéisme philosophique de Xénophane de Colophon, un contemporain du Deutéro-Isaïe et d'Ezéchiel. Dans la Bible, nous ne rencontrons cette forme de monothéisme que chez les prophètes exiliques et post-exiliques, c'est-à-dire chez le DeutéroIsaïe, Jérémie, Ezéchiel, Zacharie, Daniel et les autres. Mais tout cela n'a plus rien à voir avec Moïse. C'est pourquoi le concept d'une distinction mosaïque entre religion vraie et religion fausse est une construction erronée. S'il fallait lui trouver un nom, il faudrait la qualifier de distinction deutéro-isaïque ou jérémienne. Mais il manque dans ce cas la forme caractéristique de la violence éliminatrice qui est associée au mythe de l'Exode. Le Deutéro-Isaïe et Jérémie couvrent les autres religions de dérision et de ridicule, mais ils n'appellent pas à la persécution et à la violence.

Il semblerait donc que le monothéisme de la fidélité, dont on ne peut pas nier qu'il possède une certaine affinité avec la violence à travers sa distinction entre ami et ennemi et sa sémantique agressive de guerre sainte, ait évolué au cours de l'exil babylonien et du Second Temple dans la direction d'un monothéisme de la vérité. Cela correspondrait alors au mouvement d'une évolution plus générale du particularisme vers l'universalisme, tel qu'on peut aussi l'observer dans d'autres parties du monde ancien, par exemple en Perse et en Grèce, territoires avec lesquels la Judée, en tant que province du grand empire perse, était en étroite relation. En réalité, il n'en est rien. Le monothéisme universaliste de la vérité ne vient pas remplacer, mais il complète le monothéisme particulariste de la fidélité. Ce dernier ne perd rien de sa puissance de rayonnement, mais il continue à constituer le propre du monothéisme biblique. Le Dieu unique auquel croient juifs, chrétiens et musulmans reste également le seul Dieu, à côté duquel il ne peut pas y avoir d'autres dieux, et il est également le Dieu aimant et donc potentiellement jaloux, envers lequel il faut rester fidèle envers et contre tout. La vraie religion est définie ainsi comme la seule religion libératrice ou, comme l'exprimeront les chrétiens, salvatrice. Liberté et vérité coïncident désormais.

Les motifs de la libération, de l'alliance et de fidélité provenant de l'Exode n'apparaissent nulle part plus clairement que dans la scène saisissante décrite par Néhémie dans les chapitres IX et X. Néhémie y décrit comment les Juifs revenus de l'exil concluent une nouvelle alliance. D'abord, Ezra fait une lecture de la Torah au peuple entier réuni devant la Porte de l'Eau de Jérusalem, puis la 
«communauté de ceux qui reviennent de l'exil» fête pendant sept jours la fête des cabanes et continue à lire chaque jour des extraits de la Torah. Enfin, il est question d'une nouvelle alliance festive de ceux qui se sont ainsi séparés:

Le vingt-quatrième jour de ce mois, les Israélites, revêtus de sacs et la tête couverte de poussière, se rassemblèrent pour un jeûne. La race d'Israël se sépara de tous les gens de souche étrangère: debout, ils confessèrent leurs péchés et les iniquités de leurs pères. Debout, et chacun à sa place, ils lurent dans le livre de la Loi de Yahvé leur Dieu, durant un quart de la journée; pendant un autre quart, ils confessaient leurs péchés et se prosternaient devant Yahvé leur Dieu (Neh 9, 1-3).

Suit alors une prière dans laquelle on trouve une récapitulation longue et détaillée de toutes les actions salutaires de Dieu, c'est-àdire l'Exode, et une confession des péchés du peuple qui n'a pas honoré sa promesse et qui a été chassé du pays. Et l'ensemble se termine de la façon suivante:

À cause de tout cela, nous prenons un ferme engagement, et par écrit. Sur le document scellé figurent nos chefs, nos lévites et nos prêtres $(\ldots)$

Et le reste du peuple, les prêtres, les lévites, les portiers, les chantres, les «donnés », bref, tous ceux qui se sont séparés des peuples des pays pour adhérer à la Loi de Dieu, et aussi leurs femmes, leurs fils et filles, tous ceux qui ont l'âge de la raison, se joignent à leurs frères et chefs et s'engagent, par imprécation et serment, à marcher selon la Loi de Dieu, donnée par le ministère de Moïse, le serviteur de Dieu, à garder et observer tous les commandements de Yahvé notre Dieu, ses coutumes et ses lois. En particulier: nous ne donnerons plus nos filles aux peuples du pays et ne prendrons plus leurs filles pour nos fils (Neh $10,1 ; 10,29-31)$.

Dans une cérémonie festive, on prête de nouveau serment à l'alliance de la fidélité, et une «distinction mosaïque» au sens propre est accomplie à travers la dissolution des «mariages mixtes » entre hommes juifs et femmes cananéennes et par le bannissement des enfants nés de ces unions, et cela dans l'esprit d'une séparation et sacralisation telle qu'elle est prescrite dans la Torah.

\section{ÉCRITURE ET VIOLENCE}

Ce qui est nouveau dans cette phase du Second Temple, c'est l'Écriture, qui accède au rang d'une codification de la volonté divine 
et jouit désormais de l'autorité la plus haute et la plus absolue. C'est là quelque chose de totalement nouveau. Aucune autre religion de cette époque ne possédait un canon d'écritures saintes, qui exigeraient une observance inconditionnelle, c'est-à-dire leur transposition dans la réalité de la vie quotidienne. Cette Écriture est née dans le contexte de l'exil, moyennant certes l'utilisation de documents plus anciens. Elle a transformé les Juifs exilés en peuple du Livre et a permis sa survie en tant que tel. C'est ainsi que ce peuple a pu sauvegarder son identité alors qu'il n'avait ni état, ni territoire, ni temple, ni toutes les autres institutions matérielles, et revenir à Jérusalem après deux ou trois générations. Ceux qui sont revenus de l'exil sont alors confrontés à leurs compatriotes restés sur place, mais qui étaient dans l'ignorance des Écritures et avaient adopté les mœurs du pays, en s'assimilant et en épousant des filles du lieu. C'est alors seulement que la distinction mosaïque s'impose à nouveau, non pas sous le signe de la vérité, mais sous celui de la fidélité, de la pureté et de la sainteté.

La violence religieuse, c'est-à-dire la violence au nom de Dieu, qui est ici accomplie au sens d'une obligation sacrée et au prix d'incessantes plaintes et lamentations, est une violence fondée sur l'écriture. Elle est légitimée en faisant appel à un texte sacré, canonique, qui la prescrit de façon absolument inconditionnelle: «Tu ne dois conclure aucun contrat avec eux, tu ne dois pas les épargner, et tu ne dois pas les épouser. Ne donne pas ta fille à leur fils, et ne prends pas sa fille pour ton fils!». Un tel écrit n'a jamais existé avant l'exil. Nous disposions certes de textes religieux, historiques et littéraires, mais ils n'étaient pas dotés de cette autorité absolue. À leur place, il y avait les prophètes qui disaient à Israël «ce qui est bon et ce que le Seigneur exige de toi». Les prophètes qui reviennent toujours pour exhorter le peuple à plus de fidélité inconditionnelle ne le font qu'au moment de situations difficiles et de catastrophes qui se sont abattues sur le peuple. Cette temporalité est très décisive, car les prophètes sont ceux qui dans des situations traumatiques et post-traumatiques viennent rappeler la sortie d'Égypte et qui fondent leur exigence de fidélité sur le sauvetage, l'élection et la promesse de rédemption par Dieu. Dieu est le seul qui nous a un jour sauvés et il est seul à pouvoir le faire maintenant.

Durant la phase du Second Temple, c'est désormais l'Écriture et ses interprètes qui ont pris la place des prophètes. Alors que les faits 
de violence rapportés par l'Écriture doivent être considérés comme de pures fictions littéraires, des faits de violence réels sont désormais commis au nom de ces passages textuels. La dissolution des mariages mixtes et le bannissement des enfants doivent assurément être considérés comme tels. Ces prescriptions ont de toute évidence été contestées, et la proposition de dater le livre de Ruth de cette époque et d'y voir une sorte de contre-projet est une hypothèse très tentante. Il est également très probable que le mythe des patriarches n'a été composé que comme un contre-projet au mythe de l'Exode. «Les récits des patriarches», écrit le spécialiste d'anthropologie biblique Bernhard Lang, «sont un témoignage de l'humanisme hébraïque, un mouvement pacifiste et ouvert à l'étranger, rétif à toute utilisation de la violence, de l'époque de 500 avant J.-C. Avec les livres de la Genèse, Job et Ruth, il a donné des œuvres qui appartiennent désormais à la littérature mondiale, proches de la poésie idyllique $»^{13}$. Durant l'époque perse et au début de la période hellénistique naissent alors, à partir de ce canon d'Écritures, des attitudes que l'on pourrait qualifier, en utilisant une terminologie de notre époque, de «fondamentalistes». Avec la sémantique de la fidélité et de la jalousie désormais bien codifiée dans le canon, la force polarisatrice de la religion monothéiste peut s'exprimer dans ses conséquences sociales et politiques. C'est en se fondant sur les Écritures qu'apparaissent au sein du judaïsme des communautés et des tendances qui se séparent de la société et qui exigent de vivre d'après les préceptes de l'Écriture selon une force et une pureté particulières. C'est à ces mouvements qu'appartiennent également, à côté des Saducéens et des Pharisiens, des Esséniens et de la secte de Qumrân, Jean le Baptiste et le début du mouvement de Jésus. $\mathrm{Au}$ fondement de tous ces mouvements, on trouve toujours le même désir de séparation et de sacralisation, c'est-à-dire le motif fondamental du mythe de l'Exode et du «récit mosaïque».

Le principe de cette auto-séparation est l'Écriture: la loi théologisée comme volonté divine codifiée. Ainsi peut-on lire dans la Lettre d'Aristée, qui est une auto-description du judaïsme datant de l'époque hellénistique:

Ayant donc bien considéré tout cela, dans sa sagesse, le législateur, doué par Dieu d'une science universelle, nous a entourés d'une clôture

13. B. Lang, Buch der Kriege, p. 12. 
sans brèche et de murailles de fer, pour éviter la moindre promiscuité avec les autres peuples, nous qui, purs de corps et d'âme, libres de vaines croyances, adorons le Dieu unique et puissant, à l'exclusion d'absolument toutes les créatures [...] Pour empêcher donc que le contact impur et la conversation de gens indignes ne viennent à nous pervertir, il nous a entourés de tous les côtés de prescriptions de pureté: aliments, boissons, contacts, ouïe, vue sont l'objet d'une réglementation ${ }^{14}$.

Les guerres des Maccabées sont nées à partir de cette question de la séparation: elles étaient dirigées contre un courant qui défendait l'opinion suivante: «Allons, faisons alliance avec les nations qui nous entourent, car depuis que nous nous sommes séparés d'elles, bien des maux nous sont advenus » (I Mac 1, 12). Dans ce conflit, ce sont les Maccabées qui ont vaincu. Leur lutte n'était pas seulement une résistance contre l'occupation grecque et l'assimilation forcée sous Antiochus IV Éphiphane, mais il s'agissait aussi d'une persécution de leurs propres compatriotes hellénisés et donc jugés infidèles et schismatiques, qu'ils ont menée avec une brutalité extrême en suivant l'esprit de la guerre sainte.

L'éclatement de la révolte des Maccabées vers 165 avant J.-C. est lui-même né d'une citation de l'Écriture. Alors que le grand prêtre Mattathias se refusait fermement à sacrifier une victime païenne, un Juif s'avance pour faire le sacrifice à sa place:

À cette vue, le zèle de Mattathias s'enflamma et ses reins frémirent. Pris d'une juste colère, il courut et l'égorgea sur l'autel. Quant à l'homme du roi qui obligeait à sacrifier, il le tua dans le même temps, puis il renversa l'autel. Son zèle pour la Loi fut semblable à celui que Pinhas exerça contre Zimri, fils de Salu. Mattathias se mit à crier d'une voix forte à travers la ville: «Quiconque a le zèle de la Loi et maintient l'alliance, qu'il me suive!» Lui-même et ses fils s'enfuirent dans les montagnes, laissant dans la ville tout ce qu'ils possédaient (I Mac 2, 24-28).

On trouve un cas similaire chez Judas Maccabée qui justifie ses propres actions contre les juifs hellénisés en faisant appel au Deutéronome, où il est écrit, au chapitre XIII, qu'une guerre sainte d'extermination doit être menée contre les cités juives qui ont abandonné la loi :

14. Lettre d'Aristée, 139 et 142, trad. André Pelletier, Lettre d'Aristée à Philocrate, Paris, Le Cerf, 1962, p. 170-173 (légèrement modifiée); cf. Gerhard Delling, Die Bewältigung der Diasporasituation durch das hellenistische Judentum, Berlin, Evang. Verlag-Anst., 1987, p. 9. 
Si tu entends dire que dans l'une des villes que Yahvé ton Dieu t'a données pour y habiter, des hommes, des vauriens, issus de ta race, ont égaré leurs concitoyens en disant: «Allons servir d'autres dieux », que vous n'avez pas connus, tu examineras l'affaire, tu feras une enquête, tu interrogeras avec soin. S'il est bien avéré et s'il est bien établi qu'une telle abomination a été commise au milieu de toi, tu devras passer au fil de l'épée les habitants de cette ville, tu la voueras à l'anathème, elle et tout ce qu'elle contient; tu en rassembleras toutes les dépouilles au milieu de la place publique et tu brûleras la ville avec toutes ses dépouilles, l'offrant tout entière à Yahvé ton Dieu. Elle deviendra pour toujours une ruine, qui ne sera plus rebâtie (Dt 13, 13-19).

Le problème de la violence est étroitement lié à la scripturalisation canonisante du droit comme volonté divine révélée, car c'est à cette occasion que la sémantique archaïque de la guerre sainte et du loyalisme assyrien sont également canonisés. Ce n'est que l'Écriture qui rend possible la légitimation de la violence en faisant appel à une loi divine. La question qui nous occupe n'est pas: «Est-ce que le monothéisme a rendu le monde plus cruel?», mais bien: «Est-ce que le monothéisme, en l'occurrence le récit mosaïque, a-t-il fourni de nouveaux arguments pour légitimer la violence et la cruauté ?» Il est assurément difficile de le contester. Il suffit par exemple de jeter un coup d'œil au dernier livre du médiéviste de Münster, Gerd Althoff, sous le titre de Bienheureux ceux qui persécutent: papes et violence durant le Haut Moyen Âge, pour s'en rendre compte ${ }^{15}$. On sera impressionné par la quantité d'arguments légitimant la violence que les papes des $\mathrm{XI}^{\mathrm{e}}$ et $\mathrm{XII}^{\mathrm{e}}$ siècles ont pu extraire de la Bible et en particulier de l'Ancien Testament, dans le but d'accroître leur pouvoir politique. Le titre provocateur Bienheureux ceux qui persécutent n'est pas un détournement polémique du Sermon sur la Montagne, mais une citation du Liber ad amicum de l'évêque Bonizo de Sutri. À partir de passages comme I Samuel 15 (condamnation de Saul, parce qu'il avait épargné le roi des Amalécites Agag; il sera cruellement dépecé vivant par le prophète Samuel), Exode 32 (les Lévites qui assassinent leurs propres parents et amis après leur danse autour du veau d'or), Nombres 25 (Pinhas, qui transperce son compatriote Zimri fils de Salu en plein acte d'amour avec une Madianite) et d'autres passages de la Bible, les papes et théologiens du haut Moyen Âge ont distillé une nouvelle théorie de

15. Gerd Althoff, Selig sind, die Verfolgung ausüben. Päpste und Gewalt im Hochmittelalter, Stuttgart - Darmstadt, Theiss Verlag, 2013. 
la violence. «Les conséquences de la théorie de la violence ont été les Croisades, les campagnes contre les hérétiques et l'Inquisition», conclut Althoff. C'est ainsi que le bain de sang commis lors de la conquête de Jérusalem en 1099, qui s'est soldé par soixante-dix mille victimes, a été légitimé par le pape Urbain II par un renvoi à I Samuel, 15. Dans son ouvrage Globalisation du christianisme?, Wolfgang Reinhard a montré qu'au $\mathrm{XVI}^{\mathrm{e}}$ siècle les conquistadors espagnols ont fait appel, "pour apaiser la conscience royale» devant leurs crimes, au chapitre XX du Deutéronome dans lequel est prescrite la manière de se comporter avec les cités cananéennes: ne rien laisser en vie, tout brûler ${ }^{16}$. Aujourd'hui encore, nous ne sommes pas épargnés par une telle légitimation scripturaire de la violence, dans la mesure où le corpus scripturaire légitimant la violence s'est encore considérablement agrandi depuis la genèse de l'islam. Tout comme le principe de la formation du canon, la stratégie de tirer de l' «Écriture» non seulement le droit, mais aussi l'obligation de la persécution et de la violence, est quelque chose de propre aux religions nées de la Bible comme le sont le christianisme et l'islam.

Le judaïsme rabbinique constitue la seule exception à cette évolution. Les rabbins ont compris comment il était possible d'humaniser et de marginaliser ces textes à l'aide de leurs fines techniques d'exégèse. Il ne faut pas seulement y lire un signe de leur absence de capacité à exercer de la violence dans les conditions de la diaspora, car cette stratégie de désamorçage et d'humanisation s'étend également à la figure du martyr, qui est un autre acquis de l'époque des Maccabées et qui constitue dans une certaine mesure un contrepoint à la violence religieuse. Tuer et mourir pour Dieu et la Loi relèvent du même univers, et les deux sont rendus impossibles par la halakha rabbinique. De même, les rabbins tentent de trouver une limite à la tendance à la division et à la séparation, avec leurs lourdes conséquences sociales. Le judaïsme rabbinique a compris que la violence religieuse était un problème et a tenté de la neutraliser autant que possible. Elle est restée d'autant plus intensément vivante dans le christianisme et dans l'islam, même si l'on doit bien entendu concéder que ces religions ont également

16. Wolfgang Reinhard, Globalisierung des Christentums?, Heidelberg, Universitätsverlag Winter, 2007. 
connu des moments durant lesquels elles se sont souciées de canaliser voire d'abolir la violence religieuse.

Chercher des sources possibles dans la violence religieuse et les trouver dans la Bible hébraïque - puisqu'il est question d'origine et de commencement - n'a donc rien à avoir avec un quelconque antisémitisme, comme cela m'a encore été reproché récemment par Richard Wolin ${ }^{17}$. Ces textes hébraïques forment le canon de l'Ancien Testament à partir duquel l'Église chrétienne, puis les puritains et les Boers ont pu justifier leurs propres actes de violence. D'abord, les Juifs ont été les premiers à apprendre comment manier correctement de tels textes; deuxièmement, la Bible hébraïque et en particulier le mythe de l'Exode sont une fois pour toutes également les écrits fondateurs de l'Occident chrétien; troisièmement, le problème de la violence motivée et légitimée par la religion reste hautement actuel, et il ne peut certainement pas être résolu par des interdits de pensée et des règles de discussion restrictives.

Je concède volontiers que le concept de la «distinction mosaïque » a pu être trop simpliste et porter à confusion. Une analyse plus fine des distinctions associées au mythe de l'Exode et au monothéisme de la fidélité nous a conduit au résultat que la violence ne provient pas de la distinction entre le vrai et le faux, mais entre l'ami et l'ennemi. C'est cette distinction qui est problématique dans l'espace du religieux, en particulier lorsqu'elle est associée à la représentation apocalyptique d'un jugement dernier, dans lequel Dieu règle ses comptes avec ses ennemis. On a montré qu'elle a été introduite depuis l'extérieur dans la nouvelle religion fondée sur l'obligation et la fidélité: à partir de l'idéologie étatique assyrienne et de la sémantique de la guerre sainte. Ni l'une ni l'autre n'ont leur place dans le monde d'aujourd'hui, ni dans la religion, ni dans la politique. Mon entreprise de critique n'est donc certainement pas motivée par une velléité antisémite, mais au contraire par un projet anti-fondamentaliste. L'humanité globalisée ne peut plus se permettre une lecture des Écritures Saintes qui décrèterait que les bienheureux sont ceux qui s'adonnent à la persécution. Cela ne signifie pas qu'il faille éliminer les Écritures Saintes, mais que nous devons humaniser nos pratiques de lecture. Pour cette entreprise,

17. R. Wolin, «Biblical Blame Shift». 
la manière dont le judaïsme rabbinique a traité les textes sur la violence peut précisément servir d'exemple.

À notre époque, le mythe de l'Exode a acquis une nouvelle signification. Aujourd'hui encore, il est question d'une libération de contraintes anciennes et d'entrée dans de nouvelles obligations et dans une nouvelle loi. Cette loi porte le nom des droits de l'homme, et l'Égypte dont cette loi est supposée nous libérer comprend toutes les formes d'humiliation et de privation de droits qui ont culminé durant le $\mathrm{XX}^{\mathrm{e}}$ siècle, et parmi lesquelles l'Holocauste représente une forme incomparable de crime contre l'humanité. Tout comme dans le cas de la sortie d'Égypte, c'est le souvenir de cette histoire de souffrance qui est au fondement de l'espoir et de la disposition à entrer dans de nouvelles alliances libératrices.

jan@assmanns.de

Traduit de l'allemand par Jacob Schmutz, Université Paris-Sorbonne Abu Dhabi. 